[Original paper]

Journal of the Korean Society

for Nondestructive Testing

Vol. 32, No. 3 (2012. 6)

\title{
Low Attenuation Waveguide for Structural Health Monitoring with Leaky Surface Waves
}

\author{
M. Bezdek, K. Joseph and B. R. Tittmann ${ }^{\dagger}$
}

\begin{abstract}
Some applications require structural health monitoring in inaccessible components. This paper presents a technique useful for Structural Health Monitoring of double wall structures, such as double wall steam pipes and double wall pressure vessels separated from an ultrasonic transducer by three layers. Detection has been demonstrated at distances in excess of one meter for a fixed transducer. The case presented here is for one of the layers, the middle layer, being a fluid. For certain transducer configurations the wave propagating in the fluid is a wave with low velocity and attenuation. The paper presents a model based on wave theory and finite element simulation; the experimental set-up and observations, and comparison between theory and experiment. The results provide a description of the technique, understanding of the phenomenon and its possible applications in Structural Health Monitoring.
\end{abstract}

Keywords: Leaky Surface Acoustic Waves SAWs, Double Wall Structures, Structural Health Monitoring, Low Attenuation Multilayer Waveguide, Liquid-Solid Interface, Matrix Methods, Finite Element Simulation

\section{Introduction}

A surface wave on a liquid/solid interface is well-known to radiate acoustic energy into the liquid and is therefore rapidly attenuated. In this work, we have been able to show by experiments and calculations that the proximity of another surface (layer 1 to layer 3 and layer 3 to layer 1) sustains the surface wave through long distances for layers of both plates and concentric tubes. In addition, even when the surface wave is reflected from a distant reflector, the returning wave is sustained in the multi-layer system and can be easily detected. This is apparently one of the first observations of leaky surface waves traveling over large distances, in this case over a thousand wavelengths. The effect is modeled on the basis of a cooperative phenomenon between two interfaces separated by a water layer. The effect represents a valuable result in the wave propagation of acoustic surface waves and opens the door to many applications.

The objective of this project was the study of elastic wave propagation in multi-layer systems. An elastic wave was launched from the top of the first layer (a solid), propagated through a second layer (a fluid), into a third layer (a solid); and then it propagated down the length of the third layer to an impedance boundary. A large portion of the elastic wave energy was then reflected back through the layers to the top of the first layer and was received by a sensor. The motivation for this study was the furthering of understanding of selective wave propagation over long distances. The applications range from detecting cracks through double-wall heat exchanger and doublewall pressure vessels to determining the position of industrial lead screws separated with pressurized

[Received: February 23, 2012, Revised: June 7, 2012, Accepted: June 8, 2012] The Pennsylvania State University, University Park, PA 16802, USA †Corresponding Author: BRTESM@engr.psu.edu 
and high temperature water from another wall.

In the experiments the layers were in the form of parallel plates and concentric tubes and were separated by a layer of water where the separation was held constant. An experimental study of the best angle for a longitudinal transducer on a wedge was then conducted on the plates. Theoretical predictions of the wave properties in plates for this angle and transducer were then given. Experiments were conducted to find the optimum driving instruments and parameters. The velocity of the elastic wave was then experimentally measured. The effect of grooves on the bottom of the lower plate was investigated as well as the amount of water needed. Also, experiments were conducted with a transducer and wedge that were small enough to fit in the tubes for the tube experiments. Finally, the intensity of the wave reflected off the end of the lower plate was determined as a function of several angles of misalignment.

\section{Modeling}

The propagation of sound waves in multilayered plate structures has received considerable attention in the literature [1,2]. In seismology, many natural forms of multilayered waveguides have been studied: an ice sheet floating in an ocean, a sea layer supported by a thin bottom layer of solid, the layered form of the Earth's crust itself etc. Another class of applications can be found in the field of structural health monitoring. Important research areas include ultrasonic inspection and measurement of elastic and geometrical properties of thin surface layers, diffusion or adhesively bonded structures and composites. Since multilayered waveguides involve complicated wave mechanics, the traditional experimental-based development of such inspection techniques may become a costly and lengthy process. This evokes a need for sophisticated modeling methods which can provide accurate predictions and, consequently, reduce the number of necessary physical prototypes. In this work, we compare two different approaches based on the finite element method (FEM) and the matrix methods using an example of a three-layered waveguide with a middle liquid layer. This paper is a review and an extension of the previous works by the authors [3].

The rapid progress in the performance of personal computers has been a major factor supporting the application of computationally intensive numerical techniques. FEM has been established as one of the standard methods of computer-aided engineering for a large variety of physical systems. Its advantages include a great versatility concerning the model geometry and material composition, the availability of efficient numerical solvers, and the capability of handling coupled-field problems within a single model [4]. FEM solves the governing partial differential equations with the appropriate boundary conditions numerically. Therefore, it enables an accurate computation of complex dynamic behavior, including wave propagation in isotropic and anisotropic solids, and fluids $[5,6]$. If piezoelectric structures such as ultrasonic transducers are considered for simulation, the electric field and its interaction with the mechanical field must also be taken into account. The first application of FEM to modeling of the piezoelectric effect dates back to 1970s [7]. Nowadays, advanced 2D and 3D finite element techniques for simulating piezoelectric transducers are available $[8,9]$. Consequently, FEM can be used for modeling multilayered waveguides with solid and liquid layers including the transducers applied in a real-world setup.

On the other hand, the so-called matrix methods are based on an analytical solution of the governing partial differential equations. The derivation takes advantage of the special geometry of the problem (infinite parallel layers 
supporting strain in the modeling plane only). One arrives at a matrix description of the multilayered waveguide in terms of stresses and displacements along its external boundaries. This description can be used to obtain the modal characteristics of the waveguide (dispersion curves) and its response to a given incident plane wave (reflection and transmission coefficient). If the incident wave is finite in space and time (e.g. a pulse generated by an ultrasonic transducer), a spatial and temporal Fourier decomposition of the acoustic field has to be performed, and the response of the waveguide has to be calculated for each infinite plane wave component separately. Since both the modal and the response solutions employ numerical procedures, the matrix methods represent semi-analytical techniques which may involve extensive computational complexity. However, contrary to FEM, they can also provide valuable analytical insights into the wave mechanics of multilayered waveguides. Currently, two different matrix methods are widely in use [10]. The transfer matrix method $[11,12]$ is based on propagation of boundary conditions from one layer interface to the next one by means of multiplying layer transfer matrices. On the other hand, the global matrix method [13] combines all equations into a single global matrix.

In this paper, we focus on the application of FEM to the complete waveguide system including wedge transducers used in the experimental setup. The FEM results are compared to modal solutions obtained by means of the matrix methods.

The theoretical background of the matrix methods is given in Appendix I.

\subsection{Setup of the Three-Layered Waveguide}

Guided waves along a liquid-solid interface, the so-called leaky waves, are well-known to radiate acoustic energy into the liquid. Therefore,

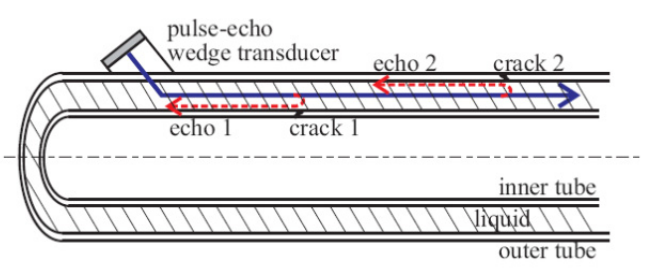

Fig. 1 Nondestructive testing of a system with concentric tubes separated by a liquid layer

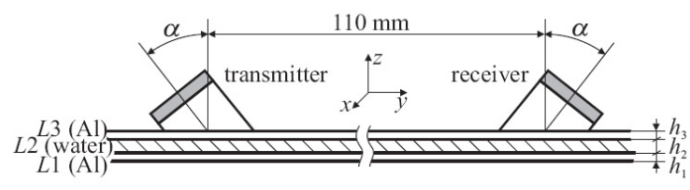

Fig. 2 Setup of the three-layered waveguide with wedge transducers

they are attenuated rapidly. However, it was demonstrated in $[14,15]$ that the proximity of another parallel solid surface may sustain the waves over distances of several meters, or a thousand wavelengths, at frequencies ranging from $1.25 \mathrm{MHz}$ to $5 \mathrm{MHz}$. In addition, it was shown that the reflected wave packet can be easily detected using a pulse-echo arrangement for systems with parallel plates or concentric tubes. This opens a range of practical applications including non-destructive testing of a double-wall heat exchanger (see Fig. 1) and position measurement of the top of a lead screw separated with pressurized liquid from a pipe wall.

The waveguide setup used in this paper (see Fig. 2) has been inspired by the work published in $[14,15]$. It consists of two aluminum plates separated by a water layer. The distance between the plates is controlled by means of a frame built of balsa wood strips. This frame also seals the water-filled space between the plates. The length of the waveguide is such that the reflections from its edges do not have to be considered in the time horizon of interest.

The waveguide is operated in throughtransmission mode. The separation between the transmitter and the receiver is $110 \mathrm{~mm}$. 
Commercial broadband piezoelectric transducers are applied. The center frequencies of the transmitter and the receiver are $1.5 \mathrm{MHz}$ and $2.25 \mathrm{MHz}$, respectively, and their fractional bandwidth reaches $50-100 \%$. The transducers are mounted on PMMA wedges providing the angle of incidence $\alpha$. Two pairs of wedges with different angles of incidence are used in experiments: $\alpha=37.0^{\circ}$ and $\alpha=53.2^{\circ}$. The coupling along the transducer-wedge and the wedge-plate contact surfaces is facilitated by means of a gel couplant.

In order to help interpret modal solutions, it is of interest to consider wave modes in the separate plates of the three-layered waveguide. Dispersion curves for plate wave modes in single-layer solid and liquid waveguides are available in the literature [16,17] (see Fig. 3). Interaction of these elementary wave modes will determine the dispersion behavior of the multilayered structure.

According to Snell's law, a wedge transducer
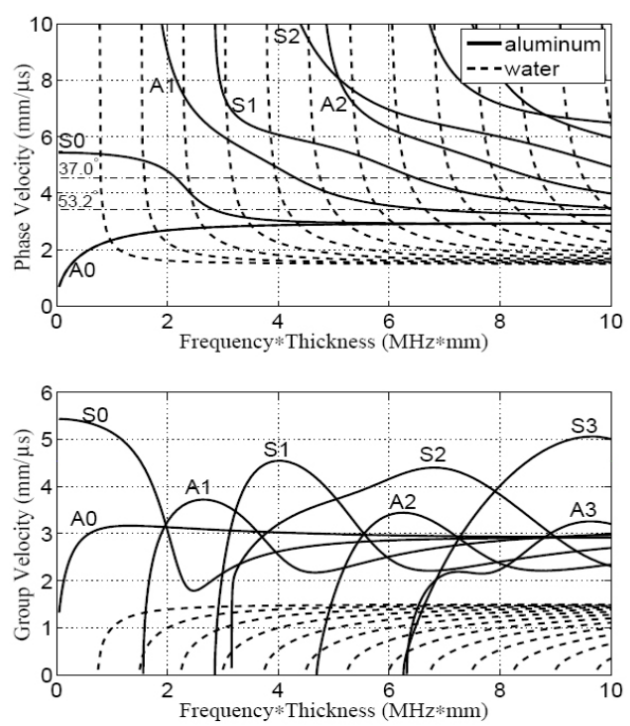

Fig. 3 Plate wave modes in a single-layer solid and liquid waveguide. Shown also are the dash-dotted activation lines for two different wedge angles $\alpha$. $\mathrm{Si}$ and $\mathrm{Ai}$ denote symmetric and antisymmetric modes in a solid plate. activates the wave mode whose phase velocity $v_{p h}$ at the given frequency is equal to

$$
v_{p h}=\frac{C_{w d g}}{\sin \alpha}
$$

where $c_{w d g}$ denotes the sound speed in the wedge $\left(c_{w d g}=2731 \mathrm{~m} / \mathrm{s}\right.$ for PMMA), and $\alpha$ is the angle of incidence. The activation lines for the two wedge angles defined above are shown in Fig. 3.

\subsection{Matrix Methods}

The derivation of the matrix methods can be found in Appendix I. Here we show the results for the three-layered waveguide computed by means of the algorithm depicted in Fig. 4.

Phase velocity dispersion curves $v_{p h}=v_{p h}(f)$ are displayed in Fig. 5. The three-layered waveguide from Fig. 2 is studied for three sample configurations of the layer thicknesses $h 1$, $h 2$ and $h 3$. For comparison, the dispersion curves of the corresponding single-layer waveguides (see Fig. 5) are shown as dashed lines.

As seen in Fig. 5, in all three cases, we notice that the overall picture closely follows the dashed pattern of modal solutions for the three separate plates of the waveguide. However, the particular dispersion curves forming the picture have different, more complicated shapes.

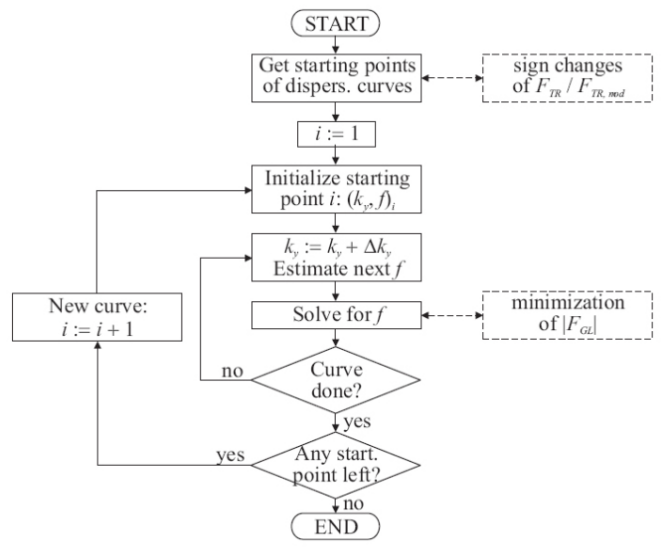

Fig. 4 Algorithm for obtaining dispersion curves 

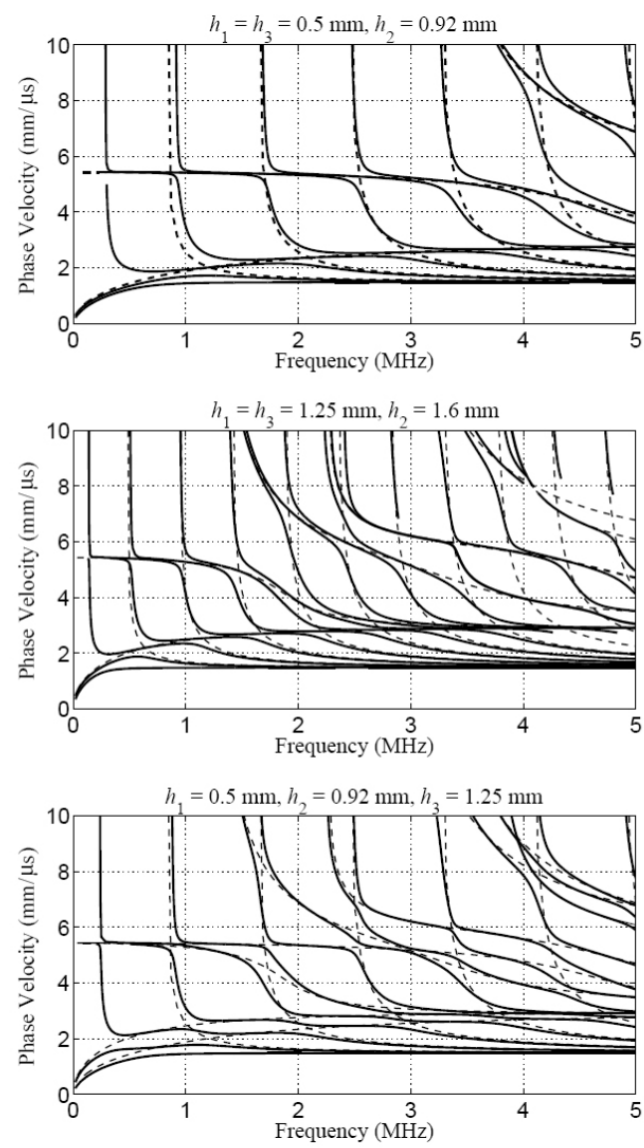

Fig. 5 Phase velocity dispersion curves for the three-layered waveguide in three different configurations. Shown also are the dashed dispersion curves of the corresponding single-layer waveguides from Fig. 2.3.

In the first and the second case, where $h 1=$ $h 3$, the curves are obviously "attracted" by the lines describing plate wave modes in the layers $L 1$ and L3. This strong tendency leads to overlapping of the dispersion curves along these lines. For this reason, curve following turns out to be difficult in these regions (see partially incomplete dispersion curves in the second case), and manual corrections are necessary. We can see that the situation is less problematic in the third case, where $h 1 \neq h 3$ and, consequently, the plate wave modes in the layers $L 1$ and $L 3$ have different characteristics.

Comparing the first and the second case, we can also conclude that, according to the expectation, the dispersion diagram is scaled in the frequency domain due to changing thicknesses of the layers. However, since there are three independent values $h 1, h 2$ and $h 3$, a single frequency-thickness parameter cannot be introduced.

\subsection{Finite Element Simulation}

\subsubsection{Model Setup}

The theoretical background of the finite element method (FEM) has been treated extensively in the literature [18-21]. A number of FEM software systems are available on the market. In this section, we perform transient FEM simulations of the complete waveguide setup using the coupled-field FEM system CAPA [22].

The experimental setup depicted in Fig. 2 makes use of finite cylindrical transducers and, therefore, requires solution in all three spatial dimensions. Nevertheless, as discussed above, the $x$-dimension is insignificant for characterization of the physical nature of the waveguide. This allows an efficient 2D FEM analysis, which can be performed on a standard PC. A portion of the $2 \mathrm{D}$ model is shown in Fig. 6.

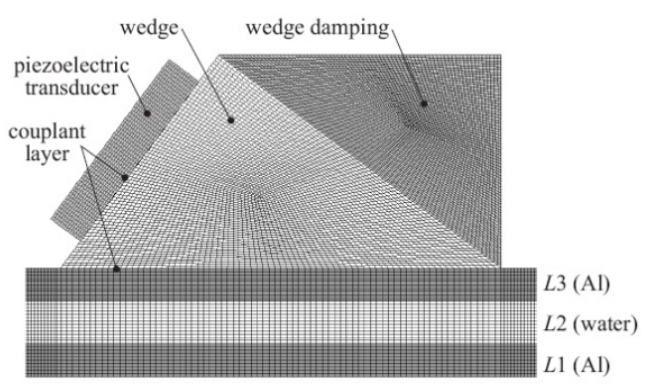

Fig. 6 Detail view of the meshed 2D FEM model of the three-layered waveguide setup. Displayed element size corresponds to a discretization rate of 4.8 elements per wavelength at $2.5 \mathrm{MHz}$ (or 12 elements per wavelength at $1 \mathrm{MHz}$ ). 
The commercial broadband transducers applied in the experimental setup are self-contained components with a complex inner structure involving various materials. The associated material and geometrical parameters are difficult to obtain and represent major uncertainty in the modeling process. For this reason, the transducers are modeled in a simplified manner as homogeneous piezoelectric elements made of the well-known ceramic PZT-5A. Their thicknesses are selected to achieve the appropriate center frequencies of the transmitter and the receiver $(1.5 \mathrm{MHz}$ and $2.25 \mathrm{MHz}$, respectively). Their bandwidth is controlled by means of manipulating the material damping parameters of PZT-5A in order to match the bandwidth of the real-world transducers. For transmitter excitation, we use a low-pass filtered pulse based on the signal of a pulser/receiver applied in the experiments. Its bandwidth is approximately $8 \mathrm{MHz}$. Nevertheless, the waveguide setup is not expected to operate efficiently at frequencies over $3 \mathrm{MHz}$ due to limited bandwidth of the transducers.

The PMMA wedges are equipped with a damping element which scatters and absorbs the energy of undesirable reflected waves. In the model, the damping element is assumed to have the same material properties as the wedge itself, but with a significantly increased material damping (by a factor of 100 with respect to the standard PMMA). Furthermore, the model includes fluid couplant layers with a thickness of $10 \mu \mathrm{m}$ along the transducer-wedge and the wedge-plate contact surfaces.

For generation of a structured 2D finite element mesh (see Fig. 6), a discretization rate of at least 12 linear elements per wavelength of the respective bulk wave (longitudinal in the wedges and the liquid, transversal in the plates) is selected, representing a compromise between model size and accuracy [23]. At a frequency of
$2.5 \mathrm{MHz}$, this discretization rate results in a model consisting of 280000 finite elements (for $h 1=h 3=1.25 \mathrm{~mm}, h 2=1.6 \mathrm{~mm}, \alpha=53.2^{\circ}$ ). A transient analysis with 9000 time steps requires about 160 minutes of computation time on a state-of-the-art PC. The effect of the discretization rate on accuracy and size of the model is further discussed in the next section.

\subsubsection{Model Validation}

In order to validate the FEM model, plate waves in the separate layer $L 3$ are considered first. In this case, the liquid layer $L 2$ and the lower plate $L 1$ are omitted from the model in Fig. 6. For each wedge angle $\alpha$, the electrical signal detected at the receiver is simulated using the broadband excitation described above. A dispersion diagram is obtained as a spectrogram of the simulated receiver signal. Since the envelope of a received signal characterizes the distribution of wave energy in time, it also carries information about dominant group velocities. Therefore, the time axis $t$ of the spectrogram can be easily mapped to the group velocity axis $v_{\mathrm{gr}}$ using

$$
v_{g r}=\frac{s}{t-\tau}
$$

where $\mathrm{s}=110 \mathrm{~mm}$ is the separation between the transmitter and the receiver, and $\tau$ is the estimated delay due to wave propagation in the wedges. In this manner, group velocity dispersion diagrams are determined here.

The simulated group velocity dispersion diagrams for two wedge angles $\alpha$ are displayed in Fig. 7. They exhibit good agreement with the available modal solution for a solid single-layer waveguide (compare to Fig. 3). In addition, they illustrate how the distribution of wave energy among the wave modes changes depending on the frequency and the wedge angle. 

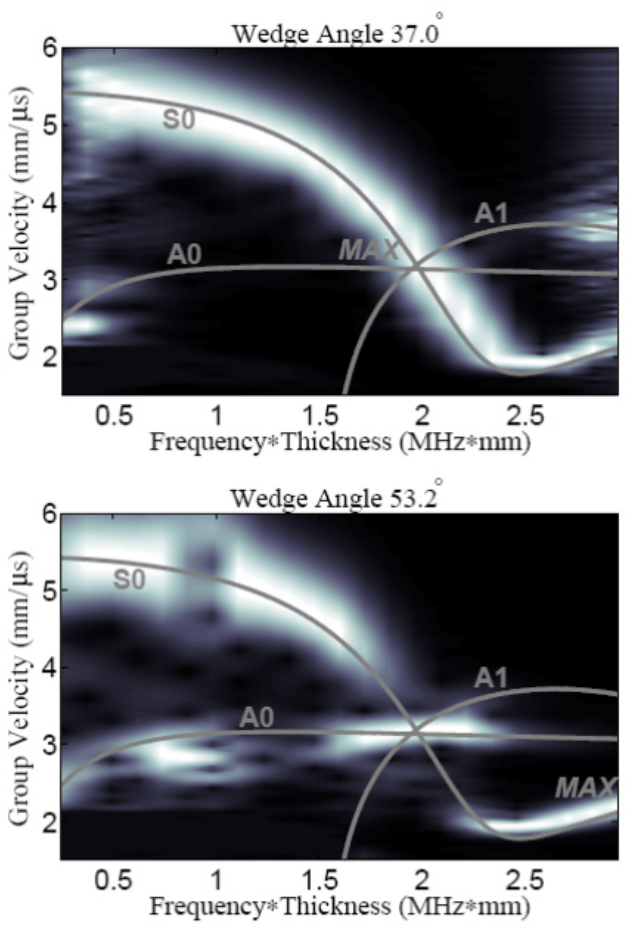

Fig. 7 Simulated dispersion diagrams with normalized amplitudes for the separate layer L3 of the waveguide setup. Shown also are the dispersion curves from Fig. 2.3 for comparison. MAX marks a dominant region where maximum amplitudes occur.

In the diagrams of Fig. 7, the spectrogram amplitude is normalized independently for every frequency, i.e. along each vertical line of a diagram. If normalization is not applied, the diagrams reveal great differences in signal amplitudes over the frequency range. It turns out that the maximum amplitude occurs at the frequency which corresponds to the intersection of the appropriate activation line given by eqn. (1) with the S0-mode curve (see Fig. 3). Thus, the simulation is, once again, in agreement with the theory. One should note that if a sufficient amount of data for various wedge angles $\alpha$ (each corresponding to one activation line, one value of $v_{p h}$ ) were available, the information about maximum amplitudes could be utilized in order to reconstruct the dispersion diagram for phase velocity $v_{p h}$.
In the second step, we perform validation for the complete three-layered waveguide using experimental results. The measured and the simulated electrical signals are processed in the same manner, allowing for a direct comparison. Fig. 8 gives an overview of the obtained dispersion diagrams for $h 1=h 3=1.25 \mathrm{~mm}, h 2$ $=1.6 \mathrm{~mm}$ and two wedge angles $\alpha$. In Fig. 8, no amplitude normalization is used, i.e. the diagrams illustrate the true distribution of wave energy in both the frequency and the group velocity domain (the normalized versions can be found in [3]). In addition, the dispersion curves calculated by means of the matrix methods (see Section 2.3.4) are shown.

First of all, we can observe satisfactory agreement between experiments and FEM simulations for the positions of dominant regions (amplitude maxima) in the dispersion diagrams. For the wedge angle $\alpha=37.0^{\circ}$, the dispersion diagram resembles the result obtained for the solid single-layer waveguide (see Fig. 7). This can be explained by the fact that the dispersion curves of the three-layered waveguide are "attracted" to the curves characterizing the layers $L 1$ and $L 3$ separately, as discussed in Section 2.3.5. Consequently, in the given frequency range and for the given wedge angle $\alpha$, the dispersion behavior of the three-layered waveguide is similar to the single-layer waveguide, although several different wave modes are involved (as opposed to the single original S0-mode). Switching of the consecutive modes manifests itself through weak vertical traces in the dispersion diagrams for $\alpha=37.0^{\circ}$. The wedge angle $\alpha=53.2^{\circ}$ leads to a completely different dispersion diagram. The original S0 and A0-mode curves are suppressed (see Fig. 7 for comparison), and a series of slowly propagating modes appear at group velocities ranging from $700 \mathrm{~m} / \mathrm{s}$ to $1700 \mathrm{~m} / \mathrm{s}$. These regions correspond well to local minima of the group velocity curves provided by the matrix methods. 

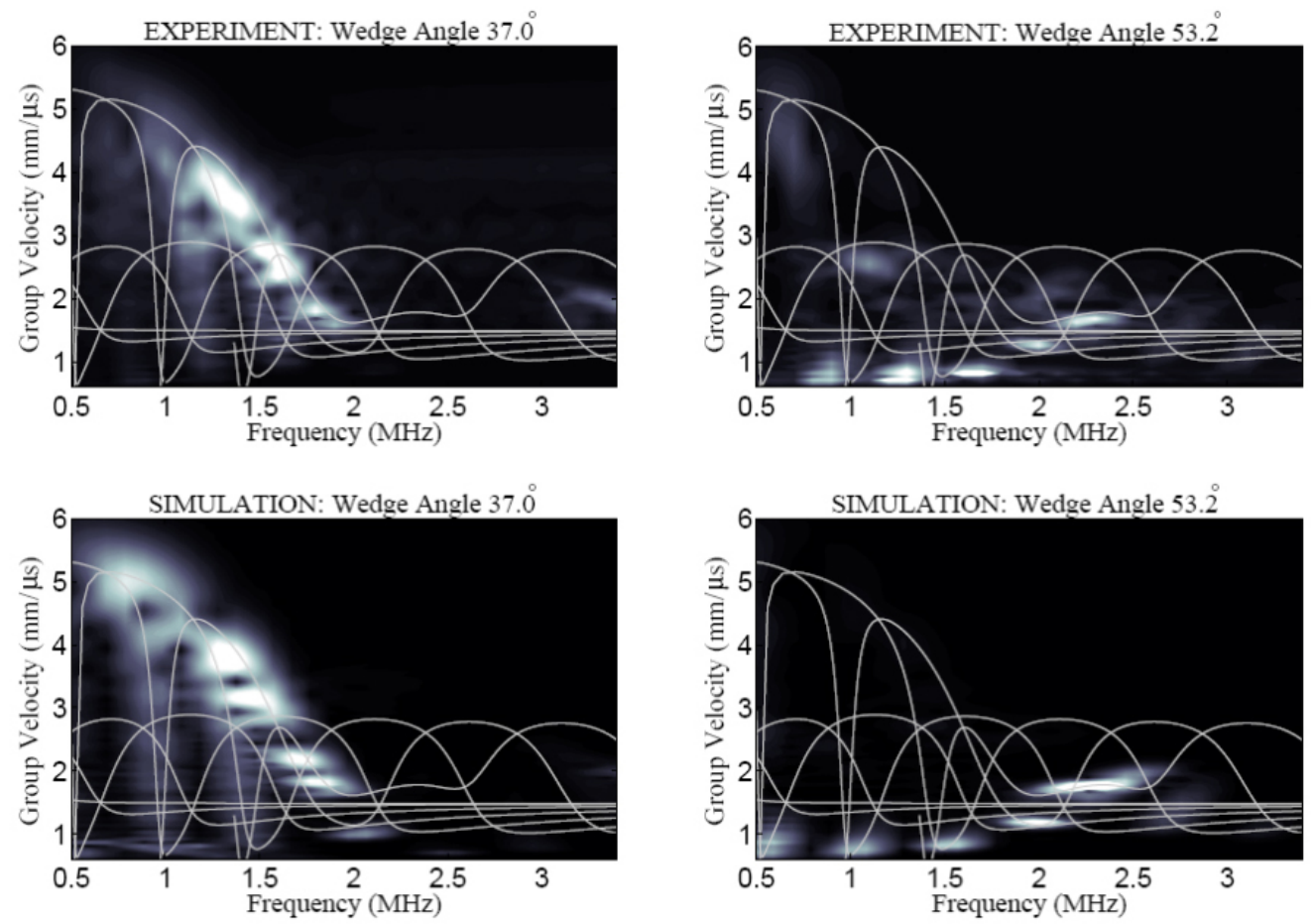

Fig. 8 Measured and simulated dispersion diagrams for the three-layered waveguide setup with $h 1=h 3=$ $1.25 \mathrm{~mm}, h 2=1.6 \mathrm{~mm}$. Shown also are the dispersion curves for the first eight modes obtained by means of the matrix methods.

Fig. 10 also shows that the dispersion curves obtained by means of the matrix methods are in excellent agreement with the FEM results, whereas discrepancies can be observed in the comparison with the experiments. This indicates that some of the parameters used by both modeling techniques may be inaccurate. Indeed, precise knowledge of the material and geometrical parameters of the system is indispensable for the success of experimental validation [23]. The accuracy of the FEM simulations is further affected by the simplified modeling of the piezoelectric transducers (including their reduction to a $2 \mathrm{D}$ space) and by spatial and temporal discretization. In order to demonstrate the effect of the discretization error, we compare envelopes of simulated receiver signals for four different values of the spatial discretization rate. The comparison is shown in Fig. 9 for the three-layered waveguide setup with $h 1=h 3=$
$1.25 \mathrm{~mm}, h 2=1.6 \mathrm{~mm}$ and $\alpha=53.2^{\circ}$. As mentioned above, the discretization rate is given as number of elements per wavelength of the respective bulk wave at a certain characteristic frequency. For simulation of the three-layered waveguide, a frequency of $2.5 \mathrm{MHz}$ is considered. We can see that a discretization rate of 3 elements per wavelength is insufficient for accurate simulation. The signal envelope is heavily distorted due to the discretization error. At a discretization rate of 6 elements per wavelength, the signal envelope gets significantly closer to the converged result. However, a slight shift along the time axis is still visible. Finally, the simulated signals at 9 and 12 elements per wavelength overlap, indicating that a further increase does not lead to an observable improvement of the accuracy. Therefore, the general discretization rate of 12 elements per wavelength used in this work is considered appropriate for the modeled system. 


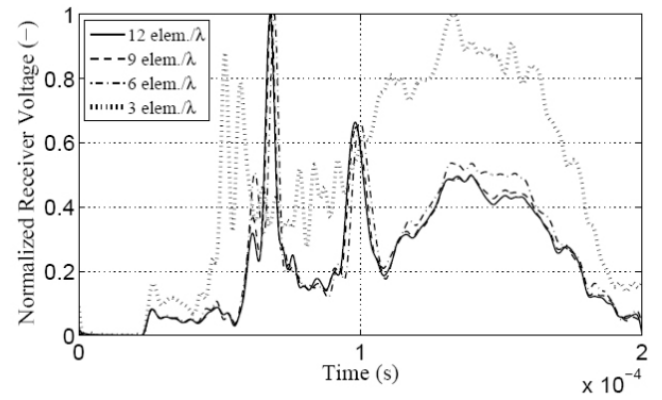

Fig. 9 Effect of the discretization rate (in elements per wavelength at $2.5 \mathrm{MHz}$ ) on the simulated signal envelope $(h 1=h 3=$ $1.25 \mathrm{~mm}, \mathrm{~h} 2=1.6 \mathrm{~mm}$ and $\alpha=53.2^{\circ}$ )

Table 1 Computational complexity of the FEM model (memory consumption of an implicit equation solver, computational time on a $2 \mathrm{GHz}$ dual-core $\mathrm{CPU}$ )

\begin{tabular}{cccc}
\hline Discretization & Elements & Memory & Time \\
\hline 3 elem. $/ \lambda$ & 19000 & $44 \mathrm{MB}$ & $1 \mathrm{~min}$ \\
$6 \mathrm{elem} . / \lambda$ & 72000 & $240 \mathrm{MB}$ & $13 \mathrm{~min}$ \\
$9 \mathrm{elem} . / \lambda$ & 159000 & $696 \mathrm{MB}$ & $55 \mathrm{~min}$ \\
$12 \mathrm{elem} . / \lambda$ & 280000 & $1529 \mathrm{MB}$ & $158 \mathrm{~min}$ \\
$15 \mathrm{elem} . / \lambda$ & 436000 & \multicolumn{3}{c}{ out of memory } \\
\hline
\end{tabular}

Table 1 summarizes the computational complexity of the reference FEM model $(h 1=$ $h 3=1.25 \mathrm{~mm}, h 2=1.6 \mathrm{~mm}$ and $\left.\alpha=53.2^{\circ}\right)$ at different discretization rates. One should note that the consumption of operational memory also depends on the selected FEM equation solver. In comparison to the implicit solver applied here, less memory-intensive solvers are available[22]. Nevertheless, they may have other disadvantages (for example, the direct explicit solver offers only conditional stability). The computational time is determined mainly by the performance of the CPU and memory subsystem.

\subsubsection{Sample Dispersion Diagrams}

After validating the FEM model, we can use it to study the influence of the geometrical configuration on the dispersion behavior of the waveguide including the wedge transducers.
Fig. 10 shows the normalized dispersion diagrams for three different sets of thicknesses $h 1, h 2, h 3$ and two different wedge angles $\alpha$. For comparison, modal solutions obtained by means of the matrix methods are provided. We can see that the FEM diagrams are in good agreement with the dispersion curves calculated by the matrix methods. The FEM simulation, however, also reveals the influence of the transducer design (here the wedge angle $\alpha$ ) on the distribution of wave energy in the frequencygroup velocity plane. For the waveguide with $h 1$ $=h 3=0.5 \mathrm{~mm}, h 2=0.92 \mathrm{~mm}$ and $\alpha=37.0^{\circ}$, most of the wave energy propagates at frequencies around $1 \mathrm{MHz}$ in a wide range of group velocities from $2500 \mathrm{~m} / \mathrm{s}$ to $5500 \mathrm{~m} / \mathrm{s}$ (see the position of amplitude maxima in Fig. 9). On the other hand, the wedge transducer with $\alpha=$ $53.2^{\circ}$ activates several consecutive modes at frequencies of $1 \mathrm{MHz}, 1.9 \mathrm{MHz}, 2.8 \mathrm{MHz}$ etc., and a nearly constant group velocity of $750 \mathrm{~m} / \mathrm{s}$. Accordingly, the two receiver signals have significantly different characteristics concerning their frequency content and travel time (envelope). In the second and the third case shown in Fig. 10, the effect of switching the values $h 1$ and $h 3(h 1=h 3)$, i.e. switching the solid layers $L 1$ and $L 3$, is examined. Obviously, the modal solutions for the two cases are identical. Nevertheless, the FEM dispersion diagrams indicate that the distribution of wave energy changes. The differences are more prominent for $\alpha=37.0^{\circ}$. For $\alpha=53.2^{\circ}$, the position of the dominant regions remains unchanged. A comparison with the first case ( $h 1$ $=h 3=0.5 \mathrm{~mm}$ ) leads to the supposition that the thickness $h 3$ determines how the energy is distributed among the waveguide modes. This can be explained by the fact that the layer $L 3$ is in contact with the transducers and may act as a "filter". The examples presented here demonstrate the potential of FEM simulation. After the validation phase of the modeling process, the 

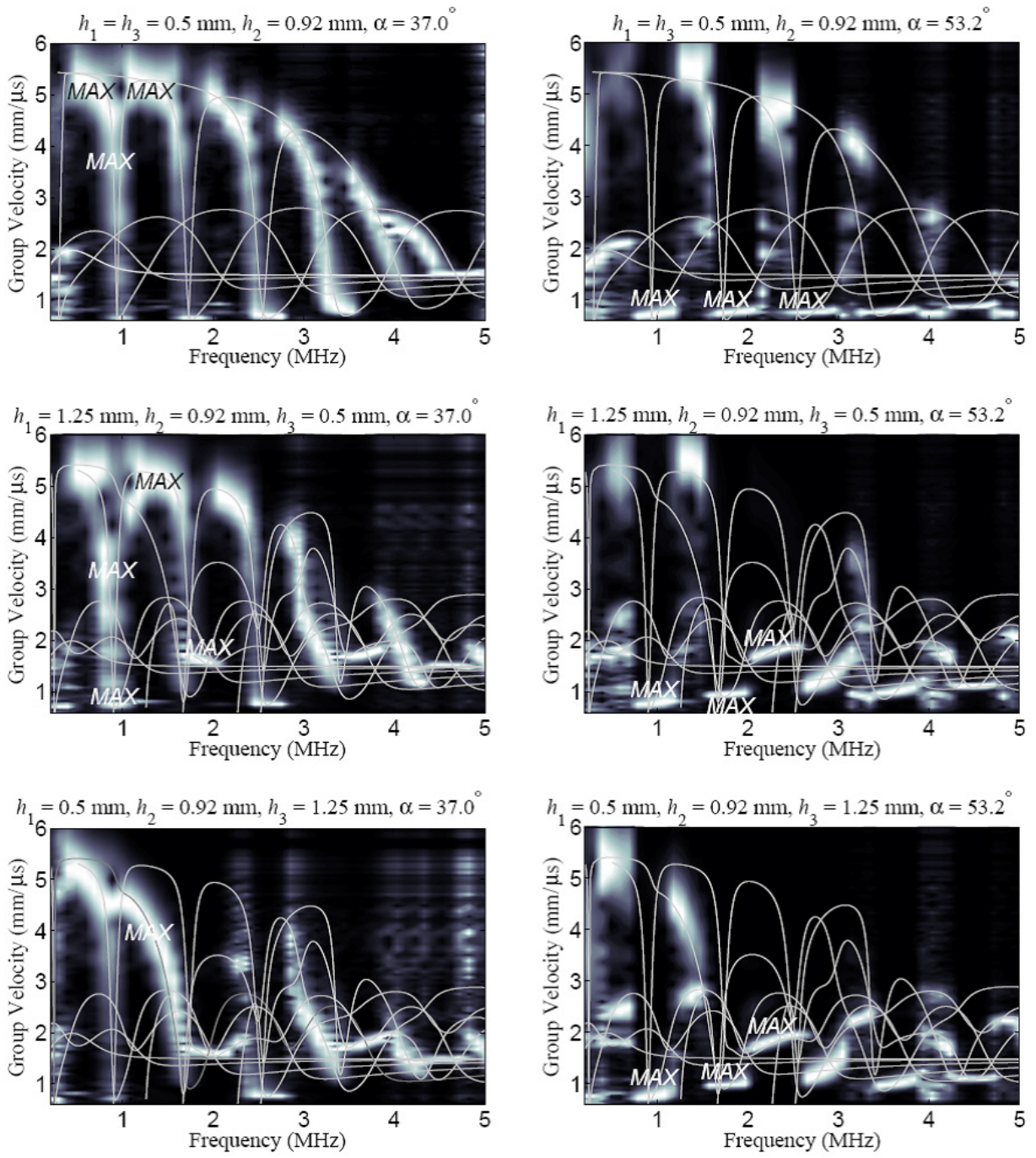

Fig. 10 Simulated dispersion diagrams with normalized amplitudes for the three-layered waveguide setup in various configurations of $h 1, h 2, h 3$ and $\alpha$. Shown also are the dispersion curves obtained by means of the matrix methods. MAX marksdominant regions where maximum amplitudes occur.
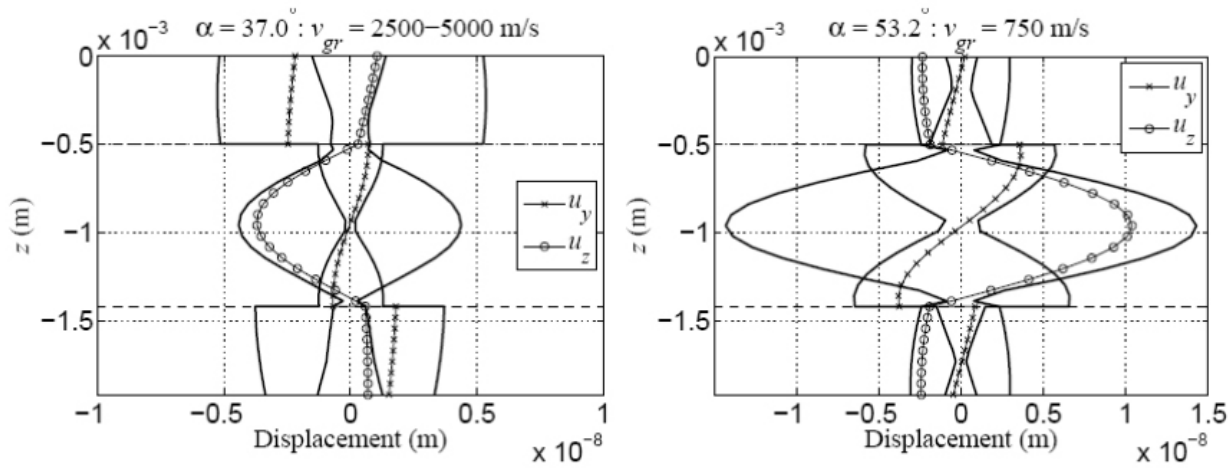

Fig. 11 Simulated displacement profiles for the three-layered waveguide with $h 1=h 3=0.5 \mathrm{~mm}, h 2=$ $0.92 \mathrm{~mm}$, center excitation frequency $\mathrm{f}=0.95 \mathrm{MHz}$, and a variable wedge angle $\alpha$. 
model parameters can be varied easily, and a large number of physical configurations can be analyzed rapidly.

\subsubsection{Displacement Profiles}

In the previous section, the FEM model has been used to compute the response of the three-layered waveguide in terms of the received electrical signal. However, the FEM simulation provides a solution of the wave field in the entire system. In this section, we utilize these data in order to depict the displacement field across the waveguide. For the analysis of displacement profiles, we consider a location half way between the transmitter and the receiver $(\mathrm{y}=0)$. We define displacement profile as a dependency of the displacements $u y, u z$ in all three layers on the $z$-coordinate (see Fig. 2). For this purpose, the origin of the z-axis $(z=$ 0 ) is assumed to be at the top of the layer $L 3$. In the solid layers $L 1$ and $L 3$, the vector displacement field $\mathrm{u}=(u y, u z)$ can be obtained directly as a result of the FEM simulation. The wave field in liquids is typically represented by a scalar field quantity, such as the acoustic pressure $p$ or the acoustic velocity potential $\Psi$. The FEM system CAPA relies on the latter formulation [22]. The potential $\Psi$ is defined by

$$
\widetilde{V}=-\nabla \Psi
$$

where $V$ is the acoustic particle velocity. Therefore, the displacement field in the liquid layer $L 2$ can be expressed in terms of $\Psi$ as

$$
u=-\int_{0}^{t} \nabla \Psi d t
$$

A discrete version of this formula can be easily implemented as a post-processing procedure.

In order to distinguish the various modes contributing to the simulated waveforms at different frequencies, we use a narrow-banded excitation signal with the center frequency of interest. Alternatively, it is possible to perform band-pass filtering of the original broad-banded waveforms. By separating a certain frequency and a time interval corresponding to the group velocity of interest, the displacement profile associated with the given region of the dispersion diagram can be obtained.

\subsubsection{Modeling Results}

Figs. 11 and 12 compare four of the cases included in Fig. 10 concerning the displacement profiles. In each case, one of the dominant regions of the dispersion diagram is characterized. Figs. 11 and 12 display maximum and minimum displacements in the given
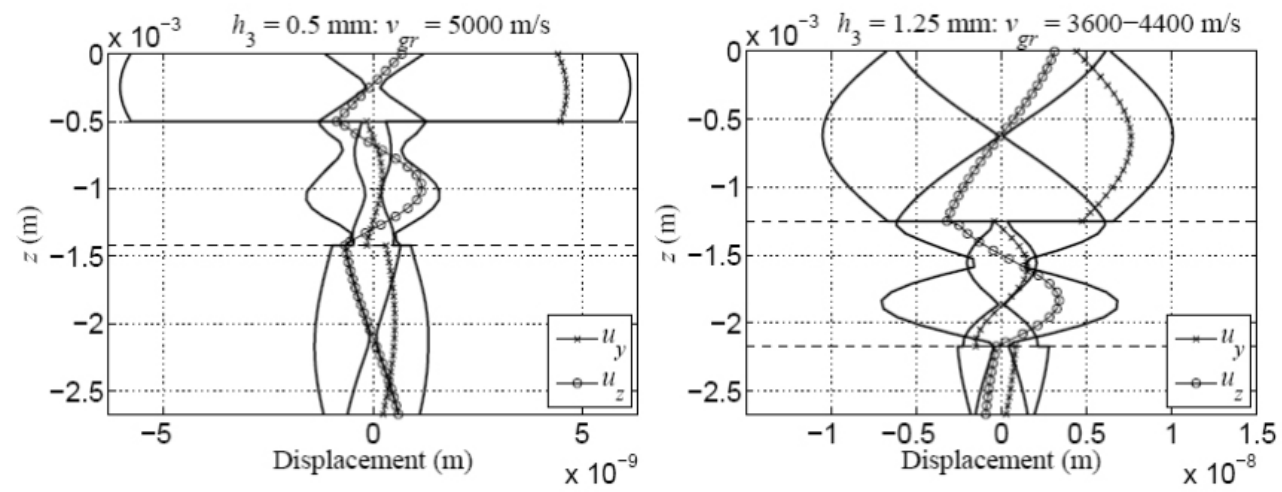

Fig. 12 Simulated displacement profiles for the three-layered waveguide with $h 2=0.92 \mathrm{~mm}, \alpha=37.0^{\circ}$, center excitation frequency $f=1.3 \mathrm{MHz}$, and $h 1, h 3$ taking the values $0.5 \mathrm{~mm}$ and $1.25 \mathrm{~mm}$ (in one or the other order). 


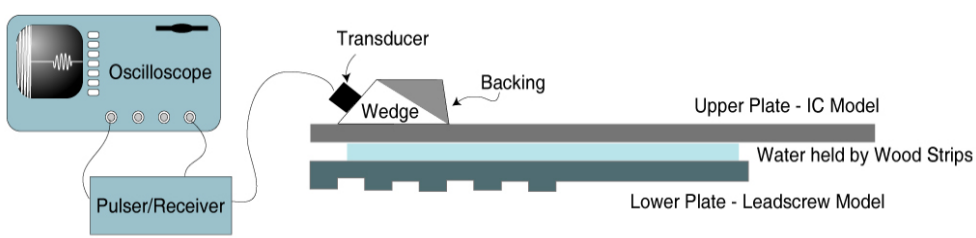

Fig. 13 Diagram of plate-on-plate setup in a pulse-echo experiment

time interval (mode shapes), as well as an instantaneous displacement profile.

We can see in Fig. 11 that the basic mode structure at $\mathrm{f}=0.95 \mathrm{MHz}$ remains similar for both wedge angles $\alpha$, although the group velocity is significantly different. However, the relative amplitude in the liquid layer $L 2$ is much higher for $\alpha=53.2^{\circ}$, i.e. the distribution of wave energy across the waveguide changes.

Fig. 12 shows more complicated displacement profiles representing higher-order modes at $\mathrm{f}=$ 1.3 MHz. In both configurations, the basic mode structure appears to be nearly identical with the exception of the $u z$-profile in the layer $L 1$. Furthermore, we can observe that the amplitudes in the top layer L3 are higher than in the bottom layer $L 1$ irrespective of its thickness. This confirms the supposition that the layer $L 3$ determines which of the existing modes is activated by the transducer. In other words, if an asymmetric waveguide is used, the receiver signal depends on which side of the waveguide the transducers are applied to.

\section{Experimental Methodology and Observations}

\subsection{System Arrangement}

The setup for the plate-on-plate experiments was as follows: a flat plate was placed on an air-cushioned table (to reduce vibrations from the room). Two parallel strips of balsa wood were then placed on the plate to provide controlled spacing between the top and the bottom plates (see Fig. 13). The space between the wood strips was filled with filtered water.
The upper plate was placed on the strips of wood. Finally, a transducer on an angled wedge was placed on top of the upper plate with coupling gel. The transducer was connected to the driver (in pulse-echo mode) and the signal from the transducer was recorded on a digital oscilloscope.

\subsection{Optimal Angle Experiments}

A commercial $1.5 \mathrm{MHz}$ transducer on a variable angle wedge was used in these experiments. When the transducer was $240 \mathrm{~mm}$ from the edge of the lower plate, the reflections from every angle were examined. To determine if a reflection was from the end of the lower plate, the upper plate and the transducer were moved relative to the lower plate. If the reflection was from the edge of the lower plate, the time of flight for the signal of the reflection would move in time. Two wedge angles were found to produce reflections from the lower plate: 35 degrees and 70 degrees. These angles correspond to the first critical angle (refracted longitudinal angle $=90$ degrees, shear $=40$ degrees) and the second critical angle (refracted shear angle $=90$ ). As seen in Figs. 13 and 14 , the reflection from the 70 degree wedge is much stronger than the reflection from the 33 degree angle wedge. Therefore, it was determined experimentally that a 70 degree in steel wedge would be the best angle to determine the position of the lower plate. Also note that there is a reflection from the end of the top plate as well as from the end of the lower plate. Note the different locations in time 


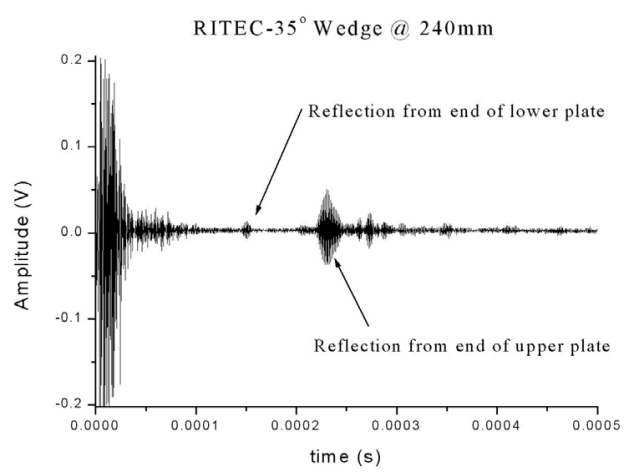

Fig. 14 Oscilloscope signal from transducer on 35 degree wedge

of each signal; this is due mode selectivity of different incident angles. The phase velocity excited is inversely dependent on the angle of incidence for guided waves in plates. Thus, the modes excited at 35 degrees are likely to be higher order modes with higher phase and group velocities then the modes likely to be excited at 70 degrees. The specific modes excited at 70 degrees will be detailed later.

\subsection{Experiments with Various Driving Instruments}

Three types of drivers (pulse generators) were examined: Panametrics, RITEC, and MATEC. The Panametrics driver produces a broad-band pulse, while the RITEC and MATEC drivers produce narrow-band tone-burst type pulses. The Panametrics and MATEC drivers produce high voltage pulses at 400 Volts, while the RITEC driver is capable of producing voltages near 1000 Volts. Experiments with the three drivers proved that the RITEC driver gave superior results and was used for the remainder of the experiments unless otherwise specified.

\subsection{Transducer Orientation}

To verify that signals were transmitted through to the lower plate, the orientation of the transducer and the lower plate with reference to the top were adjusted. For example, when the

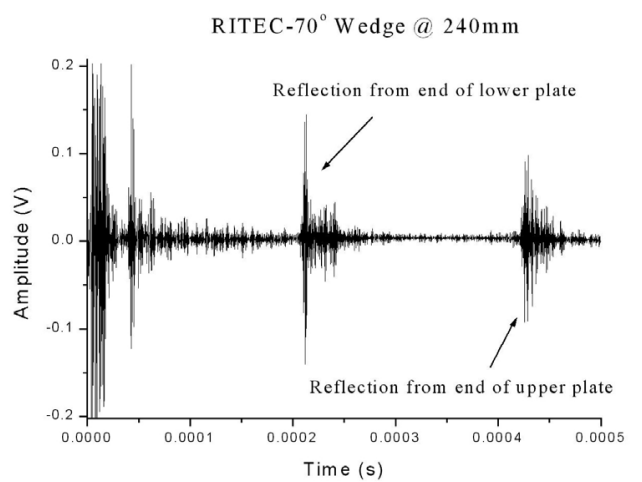

Fig. 15 Oscilloscope signal from transducer on 70 degree wedge

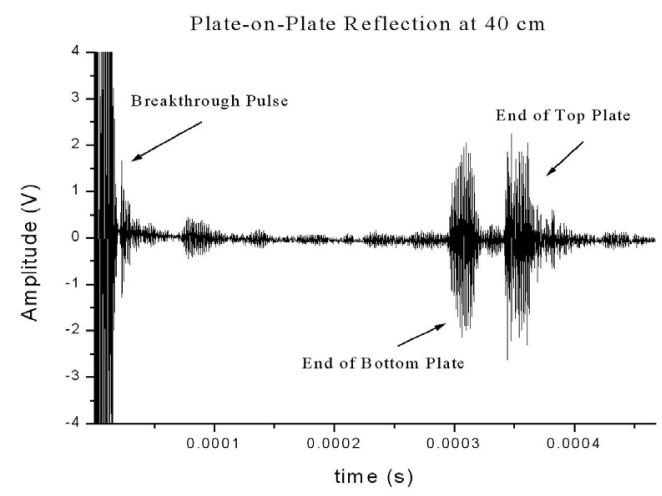

Fig. 16 Verification of ultrasonic transmission to the lower plate of the plate-on-plate system

distance of the bottom plate is increased with reference to the transducer on the top plate (which is moved slightly closer to the edge of the top plate), it can be seen that the time location of each signal moves appropriately (Fig. 15 and Fig. 16).

\subsection{Theory}

The general theory of surface and plate waves is given in [1-14]. However, the waves created with this plate-on-plate setup are a little more complex. When the angle beam transducer is set at 70 degrees angle from normal, Lamb waves are generated in the plate. Given the frequencies $(2.25 \mathrm{MHz}$ and $5 \mathrm{MHz}$ ) and angle (70 degrees) it can be shown via a dispersion diagram (Fig. 17) that the Lamb wave modes 
excited are likely to be the first symmetric and anti-symmetric modes, which happen to exhibit large out of plane particle displacements near the surfaces of the plates and have velocities very near the SAW velocity (surface acoustic wave).

The upper plate now contains plate waves that act like SAWs on both surfaces (see Fig. 18). The SAW on the lower surface of the upper plate radiates energy ( $\mathrm{L}$ waves) into the water to create surface waves in steel[3]. This radiation then hits the upper surface of the lower plate and creates a SAW on that surface. Since the lower plate is thicker, the waves on the lower plate will tend to stay on its upper surface. As long as there is water between the plates, the wave on the lower surface of the upper plate will radiate energy into the upper surface of the lower plate. Also, the SAW on the upper surface of the lower plate will radiate
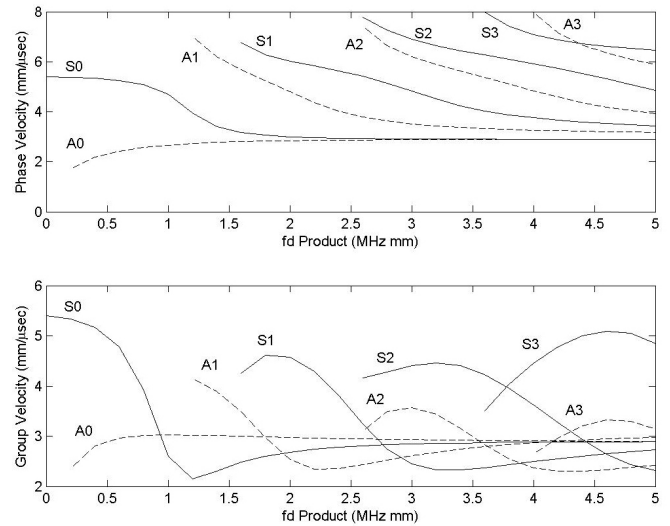

Fig. 17 Velocity dispersion diagram for Lamb wave modes in an aluminum plate

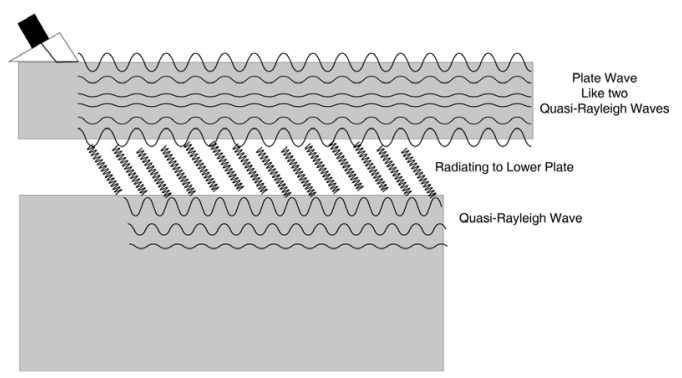

Fig. 18 Diagram of radiation pattern in plates

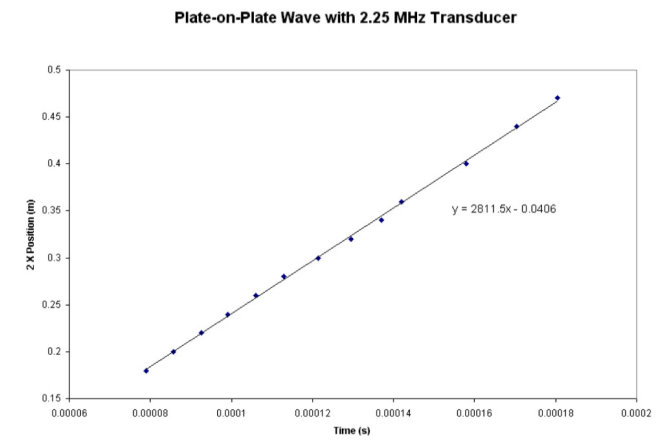

Fig. 19 Graph to determine the velocity of a plate-on-plate wave with a $2.25 \mathrm{MHz}$ transducer

energy up to the upper plate. In this way, the waves interact and create a bulk "plate-watersurface" wave. Fig. 19 is a diagram of this radiation pattern.

\subsection{Velocity Measurements}

To determine the velocity of the plateon-plate wave, the reflection from the edge of the lower plate was recorded for several positions of the transducer. For these results, the transducer was excited by the RITEC driver for a $2.25 \mathrm{MHz}$ and a $5.0 \mathrm{MHz}$ transducer with a water gap of $1 / 32 \mathrm{in}$. The total distance the pulse traversed was determined by multiplying the distance from the transducer to the edge of the lower plate by two as the pulse traveled both ways (echo mode). The time for the pulse to travel was determined by measuring the time position of the signal of the reflection from the edge of the lower plate on an oscilloscope. The velocity of the wave was then determined from the slope of the linear fit of the distance traveled versus the time of flight (see Figs. 20 and 21). The velocity was found to be 2.81 $\mathrm{km} / \mathrm{s}$ and $2.79 \mathrm{~km} / \mathrm{s}$ for the $2.25 \mathrm{MHz}$ and $5.0 \mathrm{MHz}$ transducers, respectively. If the wave was a Rayleigh wave then it should have a velocity that is approximately 0.9 times the shear velocity. The shear velocity in steel is $3.2 \mathrm{~km} / \mathrm{s}$, 


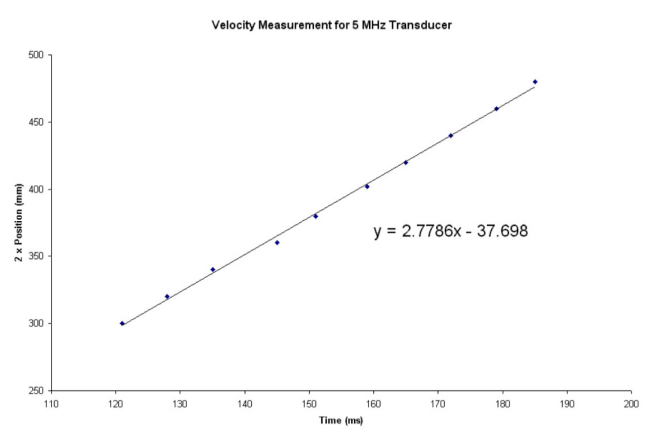

Fig. 20 Graph to determine the velocity of a plate-on-plate wave with a $5 \mathrm{MHz}$ transducer

so the Rayleigh velocity should be $2.88 \mathrm{~km} / \mathrm{s}$. As seen from the dispersion diagram, the phase velocity converges towards the Rayleigh velocity at higher fd products. The experimental setup results in lower fd products, making the expected value of the measured velocity to be slightly lower (qualitatively) then the Rayleigh velocity. The experimental results agree well with this observation.

\subsection{Water Dependence}

An interesting extension of this experiment is to determine the dependence of the plate-on-plate signal on the amount of water between the plates. If the plates are separated by air instead of water then the wave in the upper plate cannot leak into the lower plate, and there is thus no reflection from the end of the lower plate. However, the entire system does not need to be covered in water for the wave to reach the lower plate. For that reason, an experiment was conducted in which the water was restricted to a certain distance from the transducer. For this experiment the plates were placed as usual except that an extra piece of wood was placed sideways and used as a dam to limit the amount of water.

The relationship between intensity of the signal from the end of the lower plate and amount of water between the plates is as follows: as long as the wave on the lower surface of the upper plate touches water, it leaks waves into the water and thus onto the lower plate. Therefore, the reflection from the end of the lower plate should increase as the amount of water increases. However, the wave on the surface of the lower plate also leaks up to the upper plate so that the signal from the lower plate should decrease as the amount of water increases. Finally, with more water, the waves on the lower surface of the upper plate and on the upper surface of the lower plate both leak towards each other equally so that the amount of water does not affect the intensity much. In this manner the two waves reinforce each other along the propagation length.

\subsection{Angle Dependence Measurements}

Experiments were conducted to determine the dependence of the intensity of the reflection from the end of the lower plate to the symmetry of the system. The intensity dependence on angle was measured for three misalignments: top plate lifted on the side (azimuthal angle), top plate lifted on the end (longitudinal angle) and transducer turned with respect to the end of the top and bottom plates (angle of transducer). The experiments are diagramed in Fig. 21.

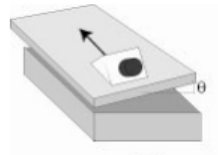

(a)

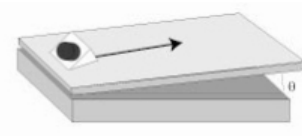

(b)

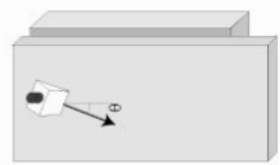

(c)

Fig. 21 Diagram of (a) azimuthal, (b) longitudinal and (c) angle of transducer in plates 


\subsubsection{Azimuthal Angle}

For this experiment, an upper plate $2.25 \mathrm{~mm}$ thick and a lower plate with a thickness of $6.6 \mathrm{~mm}$ were used. The intensity of the reflection from the end of the lower plate was measured when the plates were symmetric. An extra strip of wood was then added to the left side and the signal was once again examined. The intensity was thus measured for several angles. Finally, the signal was recorded at a very high angle to determine the amplitude of the noise. A graph of the normalized intensity (intensity minus noise divided by maximum intensity minus noise) versus angle is given in Fig. 22.

\subsubsection{Longitudinal Angle}

For this experiment, an upper plate $3.1 \mathrm{~mm}$ thick and a lower plate of $6.6 \mathrm{~mm}$ thick were used. The system was excited at $2.30 \mathrm{MHz}$. The intensity from the reflection from the end of the lower plate was measured when the system was symmetric. An extra strip of wood was then added to the bottom of the plate and the intensity was once again examined. In this case, the intensity displayed a Gaussian-like curve as expected (see Fig. 23).

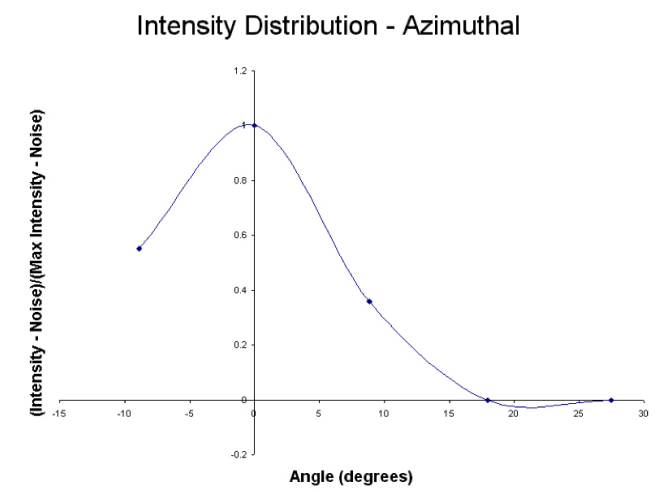

Fig. 22 Intensity distribution in plate-on-plate reflection due to changes in azimuthal angle

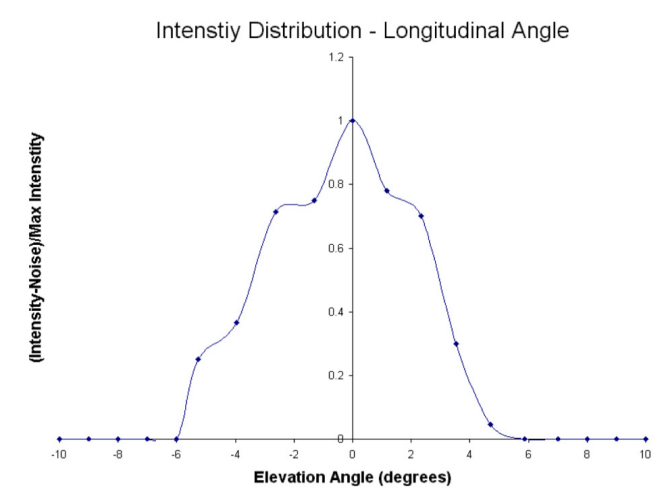

Fig. 23 Intensity distribution of plate-on-plate wave with longitudinal angle

Intensity Distribution - Angle of Transducer

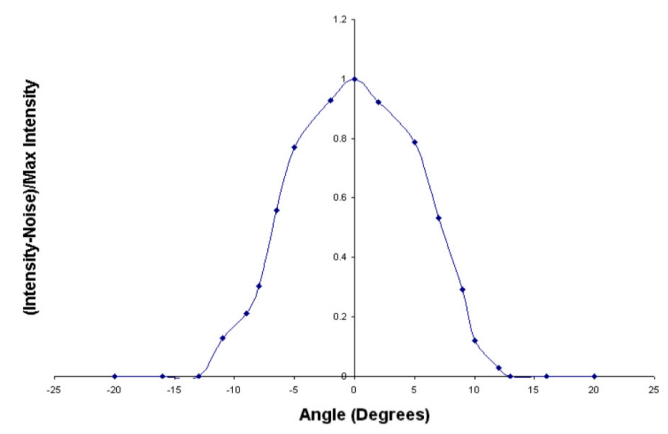

Fig. 24 Intensity distribution of plate-on-plate wave as a function of the angle of the transducer

\subsubsection{Angle of Transducer}

In this experiment, the signal was recorded as a function of the "side-to-side" angle of the transducer. The upper plate had a thickness of $2.25 \mathrm{~mm}$ and the lower plate had a thickness of $6.6 \mathrm{~mm}$. The intensity versus angle is shown in Fig. 24. The fact that this intensity decreases so quickly with angle implies that the signal in the plate is a thin ray which can be easily misaligned with the transducer.

\section{Conclusions}

This paper presents a technique useful for structural health monitoring of double wall structures, such as double wall steam pipes and double wall pressure vessels separated from an 
ultrasonic transducer by three layers. Detection has been demonstrated at distances in excess of one meter for a fixed transducer. The case presented here is for one of the layers, the middle layer, being a fluid. For certain transducer configurations the wave propagating in the fluid is a wave with low velocity and attenuation. The paper presents a model based on wave theory and finite element simulation; the experimental set-up and observations, and comparison between theory and experiment. The results provide a description of the technique, understanding of the phenomenon and its possible applications in structural health monitoring.

First, the theory of the matrix methods has been summarized. Special attention has been paid to multilayered waveguides with liquid layers. Furthermore, we have presented a numerical procedure for reconstructing dispersion curves based on the matrix methods, and we have discussed sample solutions for various configurations of the three-layered waveguide.

Second, the finite element method(FEM) has been applied to simulate the waveguide setup including real-world wedge transducers. We have introduced a 2D model of the setup and a post-processing procedure for obtaining group velocity dispersion diagrams. The model has been validated using theoretical and experimental results. In addition, the effect of discretization error has been demonstrated. In the next step, we have presented sample dispersion diagrams for two different wedge angles $\alpha$ and compared them to the modal solutions of the matrix methods. Finally, we have utilized the FEM simulation results to depict the displacement profiles (mode shapes) across the waveguide.

Third, with our experiments we have demonstrated the ability to create a plateon-plate system with guided waves capable of delivering standard ultrasonic tests to materials separated by a water layer from the transducer.
Using large angles of incidence, the first order Lamb waves are generated in the upper plate. Through experiment, the observed velocities on the upper and lower plates are expectedly similar to the velocity of a SAW, which suggests the energy leaked from the upper plate's lamb waves through the fluid layer creates SAWs on the lower plate. These SAW modes then re-contribute to the Lamb wave modes on the upper plate, sustaining the wave propagation. We have also demonstrated the stability of such a wave as well as examining the effect of grooves on the lower plate.

\section{References}

[1] W. M. Ewing, W. S. Jardetzky and F. Press, "Elastic Waves in Layered Media" New York: McGraw-Hill, (1957)

[2] L. M. Brekhovskikh, "Waves in Layered Media" New York: Academic Press, (1980)

[3] M. Bezdĕk and B. R. Tittmann, "Dispersion analysis of a three-layered waveguide with finite element and matrix methods" Acta Acoustica, Vol. 94(1), pp. 792-806 (2008)

[4] M. Kaltenbacher, "Numerical Simulation of Mechatronic Sensors and Actuators." Germany: Springer-Verlag (2004)

[5] T. J. R. Hughes, "The Finite Element Method." USA: Dover Publications (2000)

[6] O. C. Zienkiewicz and R. L. Taylor, "The Finite Element Method." UK: McGraw-Hill (1998)

[7] H. Allik and T. J. R. Hughes, "Finite Element Method for Piezoelectric Vibration," Int. J. Numerical Methods in Engineering, Vol. 2, pp. 151-157 (1970)

[8] R. Lerch, "Simulation of piezoelectric devices by two- and three-dimensional finite elements," IEEE Trans. Ultrason., Ferroelect., Freq. Contr., Vol. 37, No. 3, 
pp. 233-247 (1990)

[9] G. L. Wojcik, D. K. Vaughan, N. Abboud and J. Mould, Jr., "Electromechanical modeling using explicit time-domain finite elements," in Proc. IEEE Ultrasonics Symposium, pp. 1107-1112 (1993)

[10] M. J. S. Lowe, "Matrix techniques for modeling ultrasonic waves in multilayered media," IEEE Trans. Ultrason., Ferroelect., Freq. Contr., Vol. 42, No. 4, pp. 525-542 (1995)

[11] W. T. Thomson, "Transmission of elastic waves through a stratified solid medium," J. Appl. Phys., Vol. 21, pp. 89-93 (1950)

[12] N. A. Haskell, "Dispersion of Surface Waves on Multilayered Media," Bull. Seism. Soc. Am., Vol. 43, No. 1, pp. 17-34 (1953)

[13] L. Knopoff, "A matrix method for elastic wave problem," Bull. Seism. Soc. Am., Vol. 54, No. 1, pp. 431-438 (1964)

[14] K. L. Joseph, "Low attenuation waveguides for leaky surface waves," Master's Thesis, Pennsylvania State University, USA, (2000)

[15] K. Joseph, B. R. Tittmann, M. Pedrick and M. Kropf, "Low attenuation waveguide for leaky surface waves," in Proc. SPIE $13^{\text {th }}$ Int. Symposium on Smart Structures / NDE, San Diego, CA, USA, Feb. 26-Mar. 2, (2006)

[16] M. Redwood, "Mechanical Waveguides." USA: Pergamon Press, (1960)

[17] J. L. Rose, "Ultrasonic Waves in Solid Media." UK: Cambridge University Press, (1999)

[18] P. Cervenka and P. Challande, "A new efficient algorithm to compute the exact reflection and transmission factors for plane waves in layered absorbing media (liquids and solids)," J. Acoust. Soc. Am., Vol. 89, No. 4, pp. 1579-1589 (1991)

[19] Optimization Toolbox, The Mathworks,
USA, Available on-line: http://www. mathworks.com/products/optimization/

[20] G. E. Forsythe, M. A. Malcolm and C. B. Moler, "Computer Methods for Mathematical Computations." USA: PrenticeHall (1977)

[21] J. A. Nelder and R. Mead, "A simplex method for function minimization," Computer J., Vol. 7, pp. 308-313 (1965)

[22] R. Lerch and H. Landes, "CAPA Users Manual," Institute of Measurement Technology, University of Linz, Austria, (1995)

[23] M. Bezděk, "A Boundary Integral Method for Modeling Sound Waves in Moving Media and its Application to Ultrasonic Flowmeters," Ser. Fortschritt-Berichte VDI 8. Duesseldorf, Germany: VDI Verlag, No. 1099 (2006)

\section{Appendix I: Summary of the matrix methods}

\subsection{Basic Equations}

In this section, we briefly summarize the solution of the governing Navier equation for a multilayered structure, and we lay the basis for the matrix formulations. For a complete derivation, see for example [2].

Elastic waves in isotropic homogeneous solids are described by the Navier equation

$$
(\lambda+\mu) \nabla(\nabla \cdot \mathbf{u})+\mu \nabla^{2} \mathbf{u}=\rho\left(\partial^{2} \mathbf{u} / \partial t^{2}\right)
$$

where $\nabla$ is the nabla operator, $\mathbf{u}=\left(u_{x}, u_{y}, u_{z}\right)$ is the mechanical displacement vector, and $\lambda, \mu$ are material parameters, the so-called Lame's constants,

$$
\lambda=v E /[(1+v)(1-2 v)] \mu=E / 2(1+v),
$$

where $E$ denotes Young's modulus and $v$ the Poisson ratio. The propagation speed of bulk longitudinal waves $c_{L}$ and bulk transversal waves 
$c_{T}$ can be expressed in terms of the Lame's constants as

$$
c_{L}=\sqrt{ }(\lambda+2 \mu) / \rho_{0} \quad c_{T}=\sqrt{ } \mu / \rho_{0} .
$$

using the Helmholtz decomposition, the solution of (2) can be written as a superposition of longitudinal and transversal wave field:

$$
\mathbf{u}=\mathbf{u}_{\mathrm{L}}+\mathbf{u}_{\mathbf{T}}=\nabla \phi+\nabla \times \Psi,
$$

where the scalar potential $\phi$ and the vector potential $\Psi$ define the longitudinal and the transversal portion of the wave field, respectively. Assuming a plane wave propagation, the potentials $\phi$ and $\psi$ take the form

$$
\phi=A_{L} e^{j(\mathbf{k} \cdot \mathbf{r}-\omega t)} \quad \mathrm{I} \psi \mathrm{I}=A_{T} e^{j(\mathbf{k} \cdot \mathbf{r}-\omega \mathrm{t})}
$$

where $\mathbf{k}=\left(k_{x}, k_{y}, k_{z}\right)$ is the wave vector, $\mathbf{r}=(x, y$, $z$ ) is the position vector, $\omega=2 \pi f$ is the angular frequency, and $A_{L}, A_{T}$ denote complex-valued amplitudes of the longitudinal and the transversal plane wave, respectively. For a waveguide whose width (in the $x$-direction) is much greater than the acoustic wavelength, the solution can be further simplified by reducing it to a 2D space. In this case (the so-called plane strain), the particles are assumed to move in the modeling plane only $\left(u_{x}=0\right)$, and the strains $\varepsilon_{x x}, \varepsilon_{x y}$ and $\varepsilon_{x z}$ vanish

The matrix methods rely on the fact that a wave propagating in a multilayered waveguide can be described by superposition of elementary plane waves in each layer. It is sufficient to consider four plane waves per layer: a longitudinal and a transversal wave traveling from "down" to "up" across the waveguide (in the positive z-direction), and a longitudinal and a transversal wave traveling in the opposite direction. The amplitudes of these bulk waves are $A_{L^{+}}, A_{T^{+}}, A_{L_{-}}$and $A_{T-}$, respectively. Interaction of the elementary waves along the interfaces between adjacent layers is described by means of Snell's law. It states that the $k_{y}$-component of the wave vector remains the same for all waves on both sides of the interface. Therefore, $k_{y}$ is invariant over the entire system, and it defines the phase velocity $v_{p h}$ of the wave propagating in the waveguide:

$$
v_{p h}=\omega / k_{y}
$$

By summing the contributions of the four elementary waves according to (5), the displacement field in each layer of the waveguide can be expressed in terms of the amplitudes $A_{L^{+}}, A_{T^{+}}, A_{L-}$ and $A_{T-}$. Furthermore, the stress field $[\sigma]$ can be obtained using the strain-stress relations. For a multilayered structure, we consider those field quantities which are continuous along interfaces between adjacent layers. Along a solid-solid interface, these are the displacements $u_{y}, u_{z}$ and the stresses $\sigma_{z z}, \sigma_{y z}$. For a liquid-solid or a liquid-liquid interface, however, only the normal displacement $u_{z}$ and the normal stress $\sigma_{z z}$ remain significant. The relationships between the field quantities and the amplitudes $A_{L^{+}}, A_{T^{+}}, A_{L-}$ and $A_{T}$ - can be summarized in a matrix form by

$$
\left[\begin{array}{l}
u_{y} \\
u_{z} \\
\sigma_{z z} \\
\sigma_{y z}
\end{array}\right]=[D]\left[\begin{array}{l}
A_{L+} \\
A_{L-} \\
A_{T+} \\
A_{T-}
\end{array}\right]
$$

where the field matrix $[D]$ determines the field quantities anywhere in the multilayered structure as functions of the bulk wave amplitudes in the corresponding layer. The elements of the field matrix $[D]$ depend on the material properties of the layer, the vertical position in the layer (z-coordinate), the angular frequency $\omega$, and the wave-number $k_{y}$ (or, alternatively, the phase velocity $\left.v_{p h}\right)$. The formulae defining $[D]$ can be found in [10].

\section{The Transfer Matrix Method}

The transfer matrix method [10-12] makes use of (8) repeatedly (layer by layer) in order to eliminate the field quantities along the inner layer interfaces. In this manner, a relationship 
between the field quantities along the external boundaries of the waveguide is obtained.

Using (8), we can write for the amplitudes of the elementary waves in the bottom layer $L 1$

$$
\left[\begin{array}{c}
A_{L+} \\
A_{L-} \\
A_{T+} \\
A_{T-}
\end{array}\right]_{L 1}=[D]_{L 1, \text { bottom }}^{-1}\left[\begin{array}{l}
u_{y} \\
u_{z} \\
\sigma_{z z} \\
\sigma_{y z}
\end{array}\right]_{L 1, \text { bottom }}
$$

Substituting (9) into (8) written for the top of the layer $L 1$ leads to

$$
\left[\begin{array}{l}
u_{y} \\
u_{z} \\
\sigma_{z z} \\
\sigma_{y z}
\end{array}\right]_{L 1, t o p}=[D]_{L 1, t o p}[D]_{L 1, b o t t o m}^{-1}\left[\begin{array}{l}
u_{y} \\
u_{z} \\
\sigma_{z z} \\
\sigma_{y z}
\end{array}\right]_{L 1, \text { bottom }}
$$

Let's denote

$$
[L]_{L i}=[D]_{L i, \text { top }}[D]^{-1}{ }_{L i, \text { bottom, }}
$$

where $[L]_{L i}$ is the transfer matrix for the field quantities along the boundaries of the layer $L i$. The elements of $[L]_{L i}$ are given in an analytical form in [10].

Since the selected field quantities are continuous along the interfaces of solid layers, we can proceed with the layer $L 2$ :

$$
\left[\begin{array}{l}
u_{y} \\
u_{z} \\
\sigma_{z z} \\
\sigma_{y z}
\end{array}\right]_{L 2, \text { bottom }}=\left[\begin{array}{l}
u_{y} \\
u_{z} \\
\sigma_{z z} \\
\sigma_{y z}
\end{array}\right]_{L 1, t o p}=[L]_{L 1}\left[\begin{array}{l}
u_{y} \\
u_{z} \\
\sigma_{z z} \\
\sigma_{y z}
\end{array}\right]_{L 1, \text { bottom }}
$$

We have obtained a relationship between the field quantities at the bottoms of two adjacent layers. By repeating this process for an arbitrary number of layers $n$, we finally arrive at

$$
\left[\begin{array}{l}
u_{y} \\
u_{z} \\
\sigma_{z z} \\
\sigma_{y z}
\end{array}\right]_{L 1, t o p}=[S]\left[\begin{array}{l}
u_{y} \\
u_{z} \\
\sigma_{z z} \\
\sigma_{y z}
\end{array}\right]_{L 1, \text { bottom }}
$$

where the system matrix $[S]$ is given by ${ }^{1}$

$$
[S]=[L]_{L n}[L]_{L(n-1)} \cdots\left[L_{L 2}[L]_{L 1} .\right.
$$

In order to obtain modal solutions, we assume a multilayered structure surrounded by vacuum. Consequently, the stresses $\sigma_{z z}, \sigma_{y z}$ along the external boundaries ( $L 1$, bottom and Ln, top) vanish, and (13) becomes

$$
\left[\begin{array}{l}
u_{y} \\
u_{z} \\
0 \\
0
\end{array}\right]_{\text {Ln,top }}=[S]\left[\begin{array}{l}
u_{y} \\
u_{z} \\
0 \\
0
\end{array}\right]_{\text {L1, bottom }}
$$

In the next step, we consider the homogeneous part of this equation system

$$
\left[\begin{array}{l}
0 \\
0
\end{array}\right]=\left[\begin{array}{ll}
S_{31} & S_{32} \\
S_{41} & S_{42}
\end{array}\right]\left[\begin{array}{l}
U_{y} \\
U_{z}
\end{array}\right]_{L 1, \text { bottom }}
$$

The existence of nontrivial solutions of (16) requires that the submatrix $[S]_{3: 4,1: 2}$ is singular. The determinant of this submatrix represents the characteristic function $F_{T R}$ of the multilayered waveguide, and the characteristic equation takes the form

$$
F T R\left(k_{y}, \omega\right)=\operatorname{det}[S]_{3: 4,1: 2}=S_{31} S_{42}-S_{41} S_{32}=0 .
$$

This is an implicit equation for the angular frequency $\omega$ as a function of the wavenumber $k_{y}$. The dispersion curves $\omega=\omega\left(k_{y}\right)$ (or, alternatively, $\left.v_{p h}=v_{p h}(f)\right)$ can be obtained by means of a numerical procedure.

\section{Multilayered Waveguide with Liquid Layens}

Along a liquid-solid or a liquid-liquid interface, continuity of the normal displacement $u_{z}$ and the normal stress $\sigma_{z z}$ holds. However, the tangential displacement $u_{y}$ is not necessarily continuous, and the shear stress $\sigma_{y z}$ vanishes. Therefore, if a multilayered waveguide contains one or more liquid layers, the coupling relation (12) used above is not valid, and a modified 
matrix formulation is required [18].

Here we demonstrate the derivation for the three-layered waveguide from Fig. 2.

For the solid bottom layer $L 1$, we start with the relation (10). As explained above, the knowledge of the tangential displacement uy at the top of the layer $L 1$ is insignificant since this quantity is not transferred to the liquid layer $L 2$. Therefore, the equation for $\left\{u_{y}\right\}_{L 1, \text { top }}$ can be removed from (10). Furthermore, the liquid layer $L 2$ does not support shear stresses, i.e. $\sigma_{y z}=0$ at the top of the layer $L 1$. The same is true for the bottom of the layer $L 1$ (an external boundary) if the waveguide is surrounded by vacuum (or any fluid supporting no shear stresses). Consequently, $\{\sigma y z\}_{L 1, \text { bottom }}$ as well as the corresponding column of the transfer matrix $[L]_{L 1}$ can be omitted in (10). To sum up, we obtain

$$
\left[\begin{array}{l}
u_{z} \\
\sigma_{z z} \\
0
\end{array}\right]_{L 1, t o p}=\left[\begin{array}{lll}
L_{21} & L_{22} & L_{23} \\
L_{31} & L_{32} & L_{33} \\
L_{41} & L_{42} & L_{43}
\end{array}\right]_{L 1}\left[\begin{array}{l}
u_{y} \\
u_{z} \\
\sigma_{z z}
\end{array}\right]_{L 1, b o t t o m}
$$

In addition, the third equation of this system can be used to eliminate the unknown

$$
\begin{aligned}
\left\{\sigma_{y}\right\}_{L 1, \text { bottom }}: & \\
\left\{\sigma_{y z}\right\}_{L 1, \text { bottom }}= & -\left(1 / L_{41}\right)\left(L_{42}\left\{u_{z}\right\}_{L 1, \text { bottom }}\right. \\
& \left.+L_{43}\left\{\sigma_{z z}\right\}_{L 1, \text { bottom }}\right)
\end{aligned}
$$

and (18) becomes

$$
\left[\begin{array}{l}
u_{y} \\
\sigma_{z z}
\end{array}\right]_{L 1, t o p}=\left[\begin{array}{ccc}
L_{22}-\frac{L_{21}}{L_{41}} L_{42} & L_{23}-\frac{L_{21}}{L_{41}} L_{43} \\
L_{32}-\frac{L_{31}}{L_{41}} L_{42} & L_{33} \frac{L_{31}}{L_{41}} L_{43}
\end{array}\right]_{L 1}\left[\begin{array}{l}
u_{z} \\
\sigma_{z z}
\end{array}\right]_{L 1, \text { bottom }}
$$

The resulting modified transfer matrix (let's call it $[L]_{\text {mod,L1 }}$ )is constructed using the elements of the original $4 \times 4$ transfer matrix $[L]_{L 1}$. It describes the transfer of significant field quantities across a solid layer surrounded by liquid layers (or vacuum). If there is a subsystem of several adjacent solid layers surrounded by liquid layers, the same reasoning can be applied to the subsystem transfer matrix
$[L] L_{(i+n s o l-1)} \quad \cdots[L] L_{(i+1)}[L]_{L i}$, where $n_{\text {sol }}$ is the number of layers in the solid subsystem.

For the liquid layer $L 2$, it is necessary to reconsider the basic relation (8). Assuming that liquids do not support transversal (shear) waves, we impose $A_{T^{+}}=0$ and $A_{T-}=0$. Moreover, the equations for the insignificant field quantities $u_{y}$ and $\sigma_{y z}$ are removed. The relation (8) reduces to

$$
\left[\begin{array}{l}
u_{y} \\
\sigma_{z z}
\end{array}\right]=\left[\begin{array}{ll}
D_{21} & D_{22} \\
D_{31} & D_{32}
\end{array}\right]\left[\begin{array}{l}
A_{L+} \\
A_{L-}
\end{array}\right]
$$

where the new field matrix $[D]$ liq $=[D]_{2: 3,1: 2}$ is a submatrix of the original $4 \times 4$ field matrix $[D]$. The transfer matrix $[L] l i q, L 2$ of the liquid layer $L 2$ takes the form

$$
[L]_{l i q, L 2}=[D]_{l i q, L 2, t o p}[D]^{-1}{ }_{l i q, L 2, \text { bottom }} .
$$

The derivation for the top solid layer $L 3$ is analogous to the layer $L 1$. Finally, making use of continuity of the selected field quantities $u z$ and $O z z$, we obtain

$$
\left[\begin{array}{l}
u_{y} \\
\sigma_{z z}
\end{array}\right]_{L 3, t o p}=[S]_{\bmod }\left[\begin{array}{l}
u_{z} \\
\sigma_{z z}
\end{array}\right]_{L 1, \text { bottom }}
$$

with the modified system matrix

$$
[S]_{\bmod }=[L]_{\bmod , L 3}[L]_{l i q, L 2}[L]_{\bmod , L 1} .
$$

For modal solutions, we assume that the waveguide is surrounded by vacuum, i.e. the stress $\sigma_{z z}$ vanishes along the external boundaries:

$$
\left[\begin{array}{c}
u_{y} \\
0
\end{array}\right]_{L 3, \text { top }}=[S]_{\bmod }\left[\begin{array}{c}
u_{z} \\
0
\end{array}\right]_{L 1, \text { bottom }}
$$

The homogeneous part of this equation system

$$
0=S_{21, \text { moduz }}
$$

determines the characteristic function $F_{T R, \bmod }$ of the three-layered waveguide, and the characteristic equation is

$$
F_{T R, \bmod }(k y, \omega)=S_{21, \text { mod }}=0 .
$$




\section{The Global Matrix Method}

In contrast to the recurrent transfer matrix scheme working on the layer-by-layer basis, the global matrix method [13,10,17] describes a multilayered waveguide entirely by means of a single (global) matrix. Consequently, the dimension of this matrix depends on the number of layers $n$.

Continuity relations along the inner layer interfaces as well as the boundary conditions along the free external boundaries of the waveguide can be expressed in terms of the amplitudes $A_{L^{+}}, A_{T^{+}}, A_{L-}$ and $A_{T-}$ in the layers. For simplicity of notation, we introduce the vector $[A]_{L i}$ of bulk wave amplitudes in a layer $L i$ by

$$
[A]_{L i}=\left[\begin{array}{l}
A_{L+} \\
A_{L-} \\
A_{T+} \\
A_{T-}
\end{array}\right]_{L i}
$$

The equations for the bulk wave amplitudes in all layers can be summarized in a single equation system

$$
[G]\left[\begin{array}{l}
{[A]_{L 1}} \\
{[A]_{L 2}} \\
\vdots \\
{[A]_{L n}}
\end{array}\right]=\left[\begin{array}{c}
0 \\
0 \\
\vdots \\
0
\end{array}\right],
$$

where $[G]$ denotes the global matrix. We note that its dimension is $4 n \times 4 n$, i.e. for a solid three-layered waveguide, it consists of $12 \times 12=$ 144 elements. Nevertheless, many of them are identically zero. All nonzero elements of the matrix $[G]$ for a four-layered waveguide are given in [17]. An alternative derivation is presented in [10].

If the multilayered waveguide contains one or more liquid layers, (29) can be easily modified by enforcing $A_{T^{+}}=A_{T-}=0$ in these layers (removal of columns from $[G]$ ) and by discarding irrelevant continuity equations from the system (removal of rows from $[G]$ ). For the three-layered waveguide from Fig. 2, the matrix $[G]$ has a dimension of $10 \times 10$ with 48 nonzero elements.

The determinant of the global matrix $[G]$ represents the characteristic function $F G L$ of the multilayered waveguide. Modal solutions can be obtained by solving the characteristic equation

$$
F_{G L}(k y, \omega)=\operatorname{det}[G]=0 .
$$

The global matrix method provides a matrix formulation which can be easily adapted for any type of multilayered waveguide (with or without liquid layers, lossless or lossy) and for both modal and response solutions. Another advantage is its numerical robustness; it avoids the so-called large $f d$ problem observed with the transfer matrix method [10]. A disadvantage is the large dimension of the global matrix $[G]$ which may lead to higher computational complexity of the method. We have found that for the three-layered waveguide from Fig. 2 and the frequency range of interest, the two matrix methods discussed here offer comparable accuracy and efficiency. 